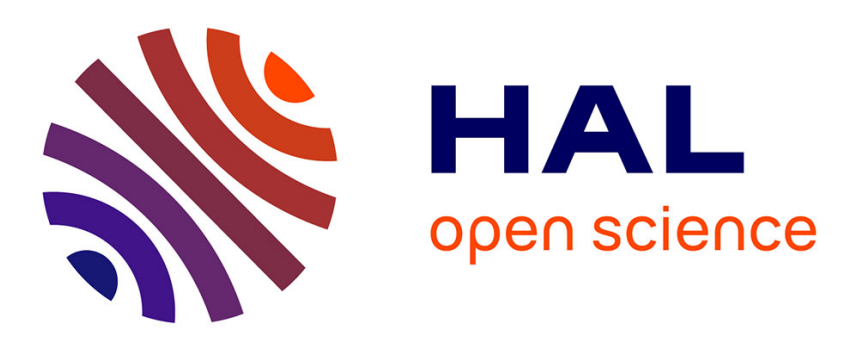

\title{
Les missions Chetwode et Vincent pendant la guerre civile espagnole : un exemple d'inflation et de concurrence humanitaire ?
}

\author{
Nathan Rousselot
}

\section{- To cite this version:}

Nathan Rousselot. Les missions Chetwode et Vincent pendant la guerre civile espagnole : un exemple d'inflation et de concurrence humanitaire ?. Relations internationales, 2018, n 176 (4), pp.41-53. 10.3917/ri.176.0041 . halshs-02614628

\section{HAL Id: halshs-02614628 \\ https://shs.hal.science/halshs-02614628}

Submitted on 21 May 2020

HAL is a multi-disciplinary open access archive for the deposit and dissemination of scientific research documents, whether they are published or not. The documents may come from teaching and research institutions in France or abroad, or from public or private research centers.
L'archive ouverte pluridisciplinaire HAL, est destinée au dépôt et à la diffusion de documents scientifiques de niveau recherche, publiés ou non, émanant des établissements d'enseignement et de recherche français ou étrangers, des laboratoires publics ou privés. 


\section{Les missions Chetwode et Vincent pendant la guerre civile espagnole : un exemple d'inflation et de concurrence humanitaire ?}

Face aux violences de la guerre civile espagnole et aux phénomènes de répression qui les accompagnent, les acteurs diplomatiques qui en sont témoins sont très tôt amenés à investir le champ humanitaire. Les échanges de prisonniers et d'"asilés" ayant trouvé refuge dans les ambassades et légations font partie de ces actions humanitaires qui se multiplient tut au long de la guerre. Les diplomaties française et britannique s'y montrent particulièrement actives. Elles sont, en 1938, à l'initiative de deux missions d'échange qui possèdent de nombreuses caractéristiques communes. Alors qu'elles agissent souvent de manière conjointe - la diplomatie française, qui tient à l'alliance britannique, plus souvent il est vrai - comment expliquer les démarches humanitaires similaires que mènent en paralèle ces deux pays ? Il s'agit d'observer, par l'étude des interactions entre les différents acteurs autour de ces deux missions d'échange, l'existence, ou non, d'une coordination humanitaire durant la guerre d'Espagne. Nous nous interrogerons sur ce qui peut paraitre comme une véritable inflation humanitaire, en nous penchant sur les dimensions strictement humanitaires des projets, mais aussi sur leurs motivations plus politiques. La multiplication de ces initiatives, dont les missions Chetwode et Vincent sont le reflet, est-elle le signe d'une concurrence humanitaire ? Ou bien ces missions présentent-elles une forme de complémentarité ?

Cette approche est d'autant plus nécessaire que les études consacrées jusqu'à présent aux activités humanitaires des diplomates n'ont pas donné une vision globale de ces interactions durant la guerre civile. Souvent centrées sur une approche nationale, elles examinent l'action humanitaire d'un pays en particulier, d'acteurs bien déterminés comme le Comité International de la Croix-Rouge ou une mission bien définie. Certains historiens ont eu l'ambition de proposer un panorama plus général comme Antonio Manuel Moral Roncal notamment, qui propose une synthèse sur l'asile diplomatique, mais traite assez peu 
des missions d'échange ${ }^{1}$. Avant lui, Javier Rubio avait proposé une étude conjointe de ces deux actions humanitaires, mais son ouvrage vise à réhabiliter l'action "humanitaire » de Franco, ce qui invite à revisiter la question ${ }^{2}$. Quoi qu'il en soit, dans ces deux cas, l'approche, certes plus globale, se fait du point de vue espagnol et traite finalement assez peu des interactions entre les acteurs humanitaires eux-mêmes. Or, il serait intéressant de porter sur ces missions humanitaires un regard décentré, qui ne soit ni national, ni focalisé sur un acteur, ni marqué par un point de vue strictement espagnol. C'est cette approche, qui permet de mettre en évidence les enjeux politiques et concurrentiels des actions humanitaires internationales, que nous nous proposons de mettre ici en œuvre.

\section{LES MISSIONS CHETWODE ET VINCENT : DEUX MISSIONS D'ÉCHANGES DANS L'ESPAGNE EN GUERRE}

La mission Chetwode est née d'une intervention humanitaire de l'ambassadeur de Grande-Bretagne, Sir Henry Chilton, le 12 novembre 1937. Celui-ci transmet au gouvernement du général Franco une proposition républicaine : les insurgés laisseraient sortir des régions du nord de l'Espagne récemment occupées par les forces rebelles toute personne, libre ou détenue, qui souhaiterait rejoindre la zone républicaine, contre l'engagement du gouvernement républicain de libérer les réfugiés des ambassades et légation étrangères de Madrid, ainsi que tous les militaires prisonniers. Le 23 novembre, le chef du cabinet diplomatique de Franco, José Antonio Sangróniz, rejette cette offre mais propose néanmoins un échange de prisonniers et d'" asilés", ainsi que la libre évacuation des vieillards, enfants et femmes qui souhaiteraient quitter l'une ou l'autre des zones. Il suggère en outre que ces échanges soient supervisés par un arbitre britannique. Le gouvernement espagnol accepte cette proposition le 2 février 1938 sous réserve de l'acceptation de deux conditions : l'échange doit s'appliquer à l'ensemble du territoire espagnol et respecter un critère de proportionnalité. Ces conditions sont acceptées par la partie adverse mais le projet tarde à se mettre en place. En effet, si la désignation du Field Marshall Chetwode comme arbitre est très vite acceptée par le gouvernement franquiste, les républicains cherchent à obtenir plutôt la nomination d'une commission internationale jouant un rôle de médiation plutôt que d'arbitrage pour superviser les échanges. Les pourparlers s'éternisent jusqu'à la fin du mois de juillet 1938, lorsqu'est acceptée la constitution d'une

1. Antonio Manuel Moral Roncal, Diplomacia, humanitarismo y espionaje en la Guerra Civil española, Madrid, Biblioteca Nueva, 2008.

2. Peter Anderson, "The Chetwode Commission and British diplomatic responses to violence behind the lines in the Spanish Civil War ", European History Quarterly, 2012, n 42/2, pp. 235-260, ici p. 252. Javier Rubio, Asilos y canjes durante la guerra civil española, Barcelone, Planeta, 1979. 
mission exclusivement britannique composée de trois membres et présidée par Chetwode. Celle-ci prend ses fonctions à la mi-août 1938. Pour autant, la mauvaise volonté des insurgés a limité amplement les résultats de la mission : à peine 500 personnes environ ont été échangées par chaque $\operatorname{camp}^{3}$.

La mission Vincent constitue une initiative relativement différente au départ, mais présente par la suite un certain nombre de similitudes avec la précédente. Alors que depuis le mois de novembre 1937 l'ambassade de France procède à l'évacuation des 900 Espagnols qui s'étaient placés sous sa protection au Lycée français de Madrid, se pose toujours la question de l'évacuation de quelque 550 Espagnols âgés de 18 à 45 ans ou militaires de carrière dont le gouvernement républicain refuse le départ. À la fin du mois de janvier 1938, l'ambassadeur Eirik Labonne envisage un échange entre la zone républicaine et la zone rebelle ${ }^{4}$. Il désigne Robert Vincent, un industriel du textile bien connu de la colonie française, "collaborateur bénévole " du consulat général à Barcelone, "capitaine de réserve de l'État-Major particulier de l'Artillerie " déjà à la tête de la mission d'évacuation des "asilés" de l'ambassade ${ }^{5}$. C'est lui, dès lors, qui doit diriger les échanges ${ }^{6}$. Pendant la nuit du 16 au 17 mars 1938, les " asilés » concernés sont évacués en France où ils sont internés à Chomérac en Ardèche, conformément à l'accord passé avec le gouvernement républicain espagnol. Celui-ci revendique tout d'abord un échange en bloc des 550 "asilés » contre 550 détenus aux mains des insurgés, mais, face à l'intransigeance des autorités rebelles, il finit par accepter un échange de 348 prisonniers contre 520 " asilés ${ }^{7}$. En dépit des promesses faites au gouvernement républicain, la France autorise le passage progressif de l'ensemble des «asilés » internés en zone insurgée avant même que les négociations n'aboutissent. Au début du mois de décembre 1938, le gouvernement du général Franco est encore débiteur de 123 détenus qu'il n'a toujours pas remis, contrairement aux engagements pris ${ }^{8}$. L'offensive en Catalogne quelques semaines plus tard, qui précipite la fin de la guerre, mit fin aux négociations d'échange sans que la contrepartie ait été respectée.

3. Juan Avilés Farré, Pasión y farsa. Franceses y británicos ante la guerra civil española, Madrid, Eudema, 1994, pp. 159-161.

4. Antonio Manuel Moral Roncal, op. cit., pp. 328, 344-345.

5. Centre des Archives Diplomatiques de Nantes, Consulat général de France à Barcelone, série $\mathrm{B}$, carton 101 (ci-après: CADN/72PO/2/101), dép. n 649 de Charles Pingaud, consul général de France à Barcelone, au Quai d'Orsay, Barcelone, 19 octobre 1937 ; CADN/72PO/E/14, dép. $\mathrm{n}^{\circ} 282$ de Pingaud au Quai d'Orsay, Barcelone, 9 mai 1937.

6. Archives du Ministère des Affaires Étrangères, La Courneuve (ci-après : AMAE-La Courneuve), Correspondance politique et commerciale, Z-Europe, Espagne, 187, dép. $n^{\circ} 79$ d'Eirik Labonne au Quai d'Orsay, Barcelone, 11 février 1938.

7. CADN/72PO/2/87, tél. de Robert Vincent au Quai d’Orsay, Biarritz, 21 septembre 1938 ; Antonio Manuel Moral Roncal, op. cit., p. 353.

8. Ibid., dép. $\mathrm{n}^{\circ} 570$ de Jacques Fouques-Duparc, conseiller de l'ambassade de France en Espagne, au Quai d'Orsay, Barcelone, 4 décembre 1938. 


\section{LE PROJET DE MÉDIATION POLITIQUE DE LA MISSION CHETWODE, OBJET D'UNE CONCURRENCE FRANCO-BRITANNIQUE?}

Très tôt, les fonctionnaires du Foreign Office se réjouissent de la contreproposition de Sangróniz qui suggère la désignation d'un arbitre, de préférence britannique, pour juger les cas litigieux lors de l'échange de prisonniers envisagé. Ils voient cette action humanitaire comme l'occasion d'accroître le prestige de la Grande-Bretagne en Espagne mais aussi sur la scène internationale, et envisagent pour celle-ci des développements politiques. Ainsi, dès le 24 janvier 1938, William Montagu-Pollock, sous-secrétaire d'État au Foreign Office, chargé des questions espagnoles, en souligne-t-il l'intérêt :

Son premier avantage sera que le Gouvernement de Sa Majesté retirera des lauriers de cet important développement, et, peut-on espérer, obtiendra avec le temps la gratitude des Espagnols; et son second avantage sera de placer le Gouvernement de Sa Majesté en position, finalement et si le moment parait opportun, de prendre l'initiative au sujet de la médiation politique 9 .

C'est cette finalité politique qui pousse les diplomates britanniques à envisager la nomination d'un médiateur d'une "certaine renommée ", capable de s'exprimer avec la «plus grande autorité » et de mener les négociations si elle évolue vers une médiation. Dans un premier temps, ils envisagent même de nommer Winston Churchill ${ }^{10}$. C'est finalement la nomination du Field Marshall Philip Chetwode qui est retenue par le nouveau secrétaire d'État aux Affaires étrangères, Lord Halifax ${ }^{11}$. Pour autant, le Foreign Office entend garder secrète la visée politique de son action humanitaire, comme le souligne son chef en présentant le projet à Chetwode à qui il demande d'accepter sa nomination :

Je faisais référence plus haut à la possibilité que ces négociations pavent la voie vers une forme de médiation politique. L'idée de médiation est pour le moment, selon nos informations, impopulaire des deux côtés, et nous sommes par conséquent particulièrement soucieux de ne pas donner l'impression que notre présente initiative a ce second objectif en vue, de peur que l'arbitre ne perde ainsi la confiance de l'un ou des deux partis en lutte ${ }^{12}$.

Mais la discrétion recherchée par le Foreign Office tient aussi à la volonté des Britanniques de mener seuls ces négociations qui, espèrent-ils, mèneront à une médiation politique dont la Grande-Bretagne seule récolterait le prestige. Ainsi, George Mounsey, secrétaire adjoint, considère-t-il le 10 février que "ce serait une erreur d'inclure [la Société des Nations] » dans le projet d'échange, et souhaite-t-il qu'il demeure dans les mains des

9. National Archives, Foreign Office, Londres, 371-22603, feuillets W831/9/41 (ci-après NA/FO 371-22603, W831/9/41). Les citations extraites d'ouvrages, articles ou documents en anglais sont traduites par nos soins.

10. NA/FO 371-22604, W1680/9/41

11. NA/FO 371-22605, W2188/9/41

12. Ibid., lettre de Lord Halifax au maréchal Chetwode, Londres, 28 février 1938 
responsables britanniques ${ }^{13}$. Par ailleurs, Mounsey et Montagu-Pollock défendaient la nomination d'une personnalité de rang plus modeste, par exemple le général Archibald Jack, par souci de discrétion et surtout afin de ne pas donner l'impression aux Français, qu'une médiation politique en Espagne intéresse aussi, que la Grande-Bretagne agit "sans eux sur un champ d'action majeur $»^{14}$. Il s'agit de dissimuler aux Français comme aux autres pays les perspectives politiques inhérentes à ce patronage humanitaire sur les échanges de prisonniers.

En fait, Labonne a vent des intentions britanniques par le correspondant de l'agence Havas à Barcelone qui l'informe que «l'Angleterre enverrait en Espagne ou en France une personnalité chargée de mettre en ouvre un vaste système d'échanges politique. " Le choix de cette personnalité « révèlerait l'importance que le Gouvernement britannique attacherait à cette mission ", et laisse penser que "sous couvert d'échanges politiques, il s'agirait peut-être en réalité d'un certain effort de médiation ${ }^{15}$. C'est en réponse aux démarches prescrites par le ministre des Affaires étrangères, Georges Bonnet, désireux d'" être renseigné sur la portée de la mission qui aurait été confiée à Sir Philip Chetwode $"^{16}$, que le Foreign Office informe Charles Corbin, l'ambassadeur de France à Londres, du projet d'échange de prisonniers en Espagne :

Le gouvernement britannique compte bien entendu mettre le gouvernement français au courant, en temps utile, de tous les détails concernant cette mission et effectuer à cet effet une démarche officielle à Paris. Il n'a pas cru devoir nous en informer plus tôt parce que les négociations dont elle est l'objet ont été fort compliquées et ont semblé plus d'une fois vouées à un échec certain. [...]

Le Foreign Office a tenu à préciser qu'en tout cas il ne saurait être question d'interpréter la mission de Sir Philip Chetwode comme une tentative de médiation entre les deux camps espagnols. Sa tâche se borne strictement à trancher les différents qui peuvent se produire à propos de l'échange des prisonniers ${ }^{17}$.

Le gouvernement britannique se garde bien de dévoiler à son homologue français les motivations sur le long terme de son projet. C'est là encore le signe qu'il entend mener ces négociations et en récolter seul les bénéfices.

Or, le gouvernement républicain refuse à la fin du mois d'avril la nomination de Chetwode comme unique arbitre et propose la formation d'une commission internationale chargée de superviser les échanges. Cette contreproposition serait due, selon le chargé d'affaires britannique John Leche, à l'influence de Labonne qu'il présente comme "un intrigant».

13. NA/FO 371-22604, W1680/9/41.

14. NA/FO 371-22605, W2188/9/41. Sur l'intérêt français pour la médiation : Nathan Rousselot, "Un diplomate face à la guerre civile espagnole : l'ambassade d'Eirik Labonne (octobre 1937octobre 1938)", Relations internationales, $n^{\circ} 170,2017$, pp. 9-23. De fait, alors que Léon Blum déclare en janvier 1938 à l'ambassadeur britannique Eric Phipps qu'à son avis, face à la question espagnole, la France et la Grande-Bretagne devraient exercer une médiation avant le printemps, le Foreign Office s'approprie l'idée mais entend la mener seul jusqu'au bout. Juan Avilés Farré, op. cit., p. 131

15. CADN, Ambassade de France à Londres, série C, carton 235, dossier 6-1-6 (ci-après : CADN/378PO/C/235, 6-1-6), tél. n²38 de Labonne au Quai d’Orsay, Barcelone, 4 avril 1938.

16. Ibid., tél. $\mathrm{n}^{\circ} 1111$ de Georges Bonnet à Charles Corbin, Paris, 13 avril 1938.

17. Ibid., tél. n 989 de Corbin au Quai d'Orsay, Londres, 14 avril 1938. 
Toujours selon lui, "la France a intérêt à y mettre son nez » et pourrait y avoir « davantage que des intérêts humanitaires ${ }^{18}$. Le lendemain, il précise avoir appris que "l'ambassadeur de France a dit aujourd'hui au ministre d'État qu'il était au courant du contenu de la note que l'ambassadeur espagnol à Londres devait présenter, et a indiqué son souhait qu'un représentant français fasse partie du Tribunal d'arbitrage $»^{19}$. Aussi le Foreign Office se retrouve-t-il bien embarrassé comme le souligne Montaigu-Pollock :

M. Leche suggère que le Gouvernement de Sa Majesté ne devrait rien avoir à faire de ces nouvelles propositions et, si je suis d'accord qu'elles sont probablement plus nocives qu'un refus définitif de négocier, je ne crois pas qu'il nous sera facile, dans les faits, de fermer entièrement la porte, en sachant en particulier que le gouvernement français est impatient d'avoir la main sur ces négociations et qu'il est susceptible de faire appel à notre coopération pour la mise en place d'un Comité international d'arbitres. [...]

Je ne crois pas improbable que le gouvernement français soit derrière le revirement du gouvernement espagnol et que nos chances de pousser ce dernier à revenir à sa décision originelle d'accepter un seul arbitre britannique dépendent de notre capacité à obtenir que les Français soutiennent notre point de vue. Le gouvernement français était sans doute enclin à suspecter que notre objectif, en proposant un arbitre britannique, était de nous mettre en position de jouer le rôle principal dans une médiation politique, et nous les avons laissés délibérément dans l'ignorance. Mais au vu des assurances catégoriques qu'ils ont reçues de notre part selon lesquelles les devoirs de Sir P. Chetwode seront limités à la mission d'échange, il ne devrait pas être difficile de convaincre le gouvernement français qu'il est de son propre intérêt d'appuyer notre initiative ${ }^{20}$

Dès le 28 avril, Eric Phipps, l'ambassadeur de Grande-Bretagne à Paris, est chargé d'inciter le gouvernement français à accepter que les négociations d'échange commencent sous les auspices du seul arbitre britannique et de demander à Labonne d'appuyer les démarches de Leche à Barcelone ${ }^{21}$. Le même jour, Alexandre Cadogan effectue une démarche similaire auprès d'Alexis Léger, le secrétaire général du Quai d'Orsay, alors en déplacement à Londres, lequel, apparemment surpris, suggère que "son gouvernement n'insisterait pas pour la désignation d'une commission française $»^{22}$. La pression des Britanniques fonctionne puisque Léger transmet quelques jours plus tard les instructions suivantes à Labonne :

Le désir manifesté par les Autorités espagnoles de voir substituer à l'activité personnelle du Maréchal Chetwode l'action d'une commission internationale soulève de vives objections de la part du gouvernement britannique qui craint notamment d'en voir résulter des délais incompatibles avec le succès de ces négociations.

18. NA/FO 371-22608, W5259/9/41, tél. nº 307 de John Leche au Foreign Office, Barcelone, 25 avril 1938

19. Ibid., tél. $\mathrm{n}^{\circ} 314$ de Leche au Foreign Office, Barcelone, 26 avril 1938

20. Ibid.

21. Ibid., tél. $\mathrm{n}^{\circ} 87$ du Foreign Office à Phipps, Londres, 28 avril 1938

22. Ibid., note d'Alexander Cadogan, 10 avril 1938. 
Le chargé d'affaires de Grande-Bretagne a reçu l'instruction d'insister à nouveau pour que le gouvernement espagnol retire les objections qu'il avait formulées contre la désignation de l'arbitre anglais unique. À la demande du Foreign Office, je vous serais obligé d'appuyer auprès du ministère d'État la demande du chargé d'affaires d'Angleterre ${ }^{23}$.

Leche avait-il raison, finalement, de voir dans la contreproposition du gouvernement républicain espagnol le résultat de manœuvres que l'ambassadeur de France à Barcelone aurait menées indépendamment du Quai d'Orsay? Certes, Labonne construit l'essentiel de son action politique autour de l'idée de médiation ${ }^{24}$; à peine informé par le correspondant de l'agence Havas du projet britannique, il écrit à ses supérieurs à Paris qu'il "serait bien important en effet que des initiatives de ce genre ou de celles suggérées dans [ses] divers télégrammes et dépêches traitant de la question ne viennent pas seulement de l'Angleterre et que ce pays seul en eût le bénéfice ${ }^{25}$. Pour autant, ce n'est pas lui qui est à l'origine du revirement espagnol. En dépit des démarches de l'ambassade de France, le gouvernement républicain maintient sa position en faveur d'une commission internationale. «Le gouvernement de Barcelone, selon le ministère d'État, avait donné son accord à la proposition anglaise initiale qui prévoyait un organisme doté de pouvoirs de médiation et éventuellement d'arbitrage, mais il ne pouvait consentir à l'octroi de pouvoirs aussi étendus à un délégué unique $^{26} »$. La proposition d'une commission internationale émane bien du gouvernement républicain qui «s'est étonné et s'inquiète un peu du titre d'arbitre donné à Sir Philip ". Les autorités espagnoles pensent " qu'un personnage aussi important n'aurait pas été désigné, ni placé à Toulouse avec un état-major important et en partie officiel, si le Foreign Office n'avait quelques vues sur les développements possibles de cette tâche ${ }^{27}$ ".

Que l'ambassadeur de France ne soit pas à l'origine du revirement ne signifie pas cependant que le chargé d'affaires britannique se trompe lorsqu'il accuse Labonne de manœuvres. Lorsque le Conseil des ministres espagnol propose que la commission soit formée d'un Britannique, d'un Américain et d'un Français ou Scandinave, Leche indique que «l'adhésion française a été réclamée par l'ambassadeur de France " et que ce dernier " a clairement intrigué, probablement de son propre fait ${ }^{28}$. Effectivement, Labonne considère que les chances de l'Angleterre d'agir comme médiateur ont diminué du côté républicain en raison de l'accord anglo-italien par lequel le gouvernement britannique reconnaît la conquête italienne

23. CADN/378PO/C/235, 6-1-6, tél. n² 214 d’Alexis Léger à Labonne, Paris, 4 mai 1938 L'ambassadeur de Grande-Bretagne à Paris en est aussitôt informé : NA/FO 371-22608, W5765/9/41, lettre de Bonnet à Phipps, Paris, 5 mai 1938.

24. Nathan Rousselot, op. cit

25. CADN/378PO/C/235, 6-1-6, tél. n² 238 de Labonne au Quai d'Orsay, Barcelone, 4 avril 1938

26. NA/FO 371-22609, W6033/9/41, lettre du Quai d'Orsay à Phipps, Paris, 9 mai 1938

27. CADN/378PO/C/235, 6-1-6, tél. n³36 de Labonne au Quai d’Orsay, Barcelone, 30 avril 1938.

28. NA/FO 371-22609, W6739/9/41, tél. nº 366 de Leche au Foreign Office, Barcelone, 26 mai 1938. 
de l'Abyssinie tandis que l'Italie s'engage à se retirer d'Espagne... après la victoire de Franco ${ }^{29}$. Il estime par conséquent que le « devoir de sollicitude de la France s'en trouve accru » et informe Bonnet que, mettant à profit les "contacts des mois passés", il "porte de [son] mieux [ses] soins vers une si désirable orientation $»^{30}$. Ainsi, c'est bien son insistance qui pousse le gouvernement espagnol à proposer d'adjoindre un Français à la future commission internationale :

Comme M. del Vayo ne suggérait pas et ne mentionnait même pas l'adjonction de membres français, je lui ai dit, après un instant de réflexion intérieure : "Vous estimez sans doute que la présence de personnalités françaises pourrait entrâner quelques complications ?" Le ministre s'en est défendu mais faiblement ${ }^{31}$.

L'attitude de l'ambassadeur déplaît au Foreign Office. Pour Leche, appuyé par ses supérieurs à Londres, il faut absolument "décliner la désignation d'un membre français car elle causerait probablement des frictions non seulement avec les Nationalistes, mais aussi avec les Républicains $»^{32}$. Dès lors, les Britanniques s'efforcent d'écarter la candidature française à la commission internationale dont ils acceptent finalement le principe, et ce d'autant plus volontiers que la candidature française ne semble pas être soutenue par Barcelone :

La possibilité d'adjoindre un membre français a été mentionnée pour faire taire l'ambassadeur de France. Il a manœuvré tout au long des négociations et a causé, je suppose, beaucoup d'ennuis au Gouvernement ${ }^{33}$.

Aussi, s'appuyant sur les réticences des républicains, le Foreign Office indique-t-il à son représentant à Burgos, Robert Hodgson, chargé de transmettre la contreproposition du gouvernement espagnol au général Franco, que cela leur "épargnerait un probable embarras, si le général Franco rejetait la suggestion d'inclure un Français " ${ }^{34}$.

Finalement, Franco rejette la désignation de membres tant français qu'américain et scandinave au prétexte qu'aucun de ces pays n'a reconnu de facto ou de jure son gouvernement. Face à sa contreproposition d'une commission internationale qui serait formée d'un membre britannique, d'un représentant d'un pays ayant reconnu son gouvernement (de préférence

29. Ángel Viñas, El Honor de la República. Entre el acoso fascista, la hostilidad británica y la política de Stalin, Barcelone, Crítica, 2009, pp. 273 sq.

30. CADN, Ambassade de France à Madrid, Série B, carton 591 (ci-après : CADN/396PO/B/591), tél. nº 410-415 de Labonne au Quai d'Orsay, Barcelone, 17 mai 1938.

31. CADN, Ambassade de France à Madrid, Série B, sous-série Guerre-Civile, carton 568, dossier 4-C3 (ci-après : CADN/396PO/B/568, GC 4-C3), tél. n 363-367 de Labonne au Quai d'Orsay, Barcelone, 26 avril 1938

32. NA/FO 371-22609, W5801/9/41, tél. nº 341 de Leche au Foreign Office, Barcelone, 6 mai 1938

33. Ibid., tél. $\mathrm{n}^{\circ} 373$ de Leche au Foreign Office, Barcelone, 30 mai 1938. Le gouvernement républicain n'a certainement pas pardonné à Labonne ses ingérences en faveur d'une médiation, à l'origine de la crise ministérielle d'avril 1938: Nathan Rousselot, op. cit.

34. NA/FO 371-22609, W6906/9/41, tél. nº 186 du Foreign Office à Robert Hodgson, Londres, 22 juin 1938. 
l'Allemagne ou l'Italie) et d'un membre choisi par le gouvernement républicain (à l'exception de l'URSS), les autorités républicaines finissent par proposer une commission exclusivement britannique composée de trois membres. C'est cette dernière proposition qui donne enfin naissance à la commission Chetwode, après de longs mois de négociations. La France s'en trouve définitivement exclue. Elle a cependant contribué, par l'intermédiaire de son représentant à Barcelone, à prolonger des tractations déjà lentes et difficiles, menaçant les efforts humanitaires du gouvernement britannique. La perspective politique de son initiative peut seule expliquer les entraves mises par la France. L'ambassadeur Labonne ne cherche pas à entrer dans une forme de concurrence humanitaire avec la Grande-Bretagne ; il entend simplement s'assurer que Paris puisse prendre part au processus de médiation et de paix en Espagne. Quant à la dimension purement humanitaire de la mission Chetwode, le gouvernement français et son ambassade semblent adopter une posture opportuniste, bien qu'elle puisse entrer par moments en concurrence de fait avec la mission Vincent.

\section{LA MISSION VINCENT FACE À LA MISSION CHETWODE ENTRE CONCURRENCE ET COMPLÉMENTARITÉ}

Parallèlement aux discussions concernant la mise en place de la mission Chetwode, Robert Vincent entame les négociations relatives à l'échange des 550 " asilés " d'âge militaire qui se trouvaient toujours sous la protection de l'ambassade. Dès le début, l'initiative britannique semble compliquer la tâche des Français. C'est que les deux missions partagent un but commun, l'échange d'individus entre les deux zones, et sont susceptibles de s'intéresser aux mêmes individus, notamment en zone rebelle. C'est ce que souligne Labonne dès la mi-février :

Parmi les asilés en état de porter les armes [...] se trouvent environ 70 officiers inscrits sur les rôles de l'ancienne armée. Comme il y a très peu d'officiers républicains en zone nationaliste, que des projets d'échanges par l'entremise anglaise sont en cours au sujet des prisonniers de guerre proprement dits, une difficulté sérieuse peut s'élever de ce fait. [...] Ce sera l'un des premiers problèmes que M. Vincent aura à traiter ${ }^{35}$.

On voit ici les conséquences de la multiplication des projets d'échanges qui peuvent, du fait de l'absence de coordination internationale, entrer en concurrence. Chaque acteur est tenté de faire aboutir son projet au détriment des autres projets existants, qu'il cherche à faire passer, dès lors qu'ils sont soumis à une quelconque difficulté, au second plan afin de ne pas compromettre sa propre initiative. Ainsi, lorsque les négociations visant à instaurer la mission d'échange britannique semblent s'éterniser

35. CADN/396PO/B/568, GC 4-C3, dép. nº 86 de Labonne au Quai d’Orsay, Barcelone, 16 février 1938 
face à la volonté républicaine d'instaurer une commission internationale, Labonne, tout en défendant sans grande énergie le point de vue britannique, s'empresse de rappeler la mission française d'échange au bon souvenir d'Álvarez del Vayo :

J'ai cru ne devoir formuler aucune observation, me bornant à dire que je donnerai aussitôt connaissance à Votre Excellence de cette note lorsqu'elle me sera communiquée.

Mais j'en ai incidemment profité pour rappeler l'affaire de Chomérac, pour représenter l'intérêt de conclure promptement au moins une affaire d'échange et de mener harmonieusement à son dernier terme une œuvre humanitaire qui fasse honneur aussi bien au gouvernement de l'Espagne républicaine qu'au gouvernement français. L'opportunité de ces considérations a été aperçue et il se peut que des décisions rapides apparaissent ${ }^{36}$.

Cette multiplication des projets d'échanges tend aussi à compliquer les négociations de part et d'autre. L'adoption d'un principe pour l'une des missions peut conduire l'un des camps à revendiquer son adoption pour un autre projet d'échange. Chacune des parties tend à maintenir une position de principe, de peur qu'une concession accordée ne le soit définitivement pour les négociations futures. C'est ce que constate en tout cas l'ambassadeur de France au sujet de la mission Vincent. Alors que les insurgés proposent d'échanger les 500 " asilés » d'âge militaire de l'ambassade contre 300 détenus, le gouvernement républicain s'attache fermement au principe d'un échange tête pour tête, 500 contre 500. Or, selon Labonne, le « résultat de ces démarches et la position de principe prise en prévision d'éventuelles tractations de la mission Chetwode ne permettent guère plus d'espérer que le gouvernement républicain abandonnera le principe tête pour tête $\|^{37}$.

Tout cela entraîne aussi un certain nombre de déconvenues lorsque les mêmes individus sont concernés par divers projets. Ainsi le gouvernement républicain rejette-t-il une liste transmise par Vincent de 70 détenus, proposés par les autorités insurgées de Burgos en échange des officiers " asilés " de l'ambassade, en signalant «que six des détenus indiqués par $\mathrm{M}$. Vincent ont déjà quitté l'Espagne nationaliste à la suite de l'échange des 147 asilés de la légation de Cuba " qui a eu lieu la veille ${ }^{38}$. D'autre part, le manque de coordination de ces missions d'échanges a pu permettre au gouvernement du général Franco de faire obstacle aux négociations et de contourner certains engagements pris, comme l'en accusent les républicains auprès de l'ambassade de France :

Le gouvernement républicain avait accepté sans discussion une liste proposée par les nationalistes de 230 membres. Pas une seule des personnes figurant sur cette liste ne lui a été cédée et les raisons invoquées de l'autre côté, à savoir que, durant

36. CADN/396PO/B/591, tél. n 427-431 de Labonne au Quai d’Orsay, Barcelone, 26 mai 1938. Sur l'affaire de Chomérac, voir plus haut.

37. Ibid., tél. n 434-435 de Labonne au Quai d'Orsay, Barcelone, 28 mai 1938

38. CADN/72PO/2/87, tél. $n^{\circ} 767-768$ de Pierre Francfort au Quai d'Orsay, Barcelone, 25 novembre 1938 
la négociation, ces personnes ont été comprises dans d'autres échanges, sont paraît-il, inexactes ; aucune en tout cas n'a fait partie d'un convoi quelconque ${ }^{39}$.

Pour autant, si les missions d'échange peuvent, de fait, entrer en concurrence, elles peuvent aussi apparaître complémentaires par d'autres aspects. Ainsi, le gouvernement français fait preuve d'une forme d'opportunisme à l'égard de la mission Chetwode, dont il entend user pour pallier les limites de la mission Vincent. Alors que la mission française ne prévoit pas d'inclure les ressortissants français détenus par les forces du général Franco, le Quai d'Orsay suggère de profiter de l'instauration de la mission britannique pour obtenir leur libération. Il estime qu'il « conviendrait que les ressortissants français prisonniers en zone insurgée ne fussent pas omis dans l'éventualité d'un échange " ${ }^{40}$.

Se pose aussi la question des Espagnols qui, malgré les évacuations, sont restés à Madrid dans les hôpitaux français sous la protection de Paris et dont le gouvernement du général Franco demande l'échange. Or, comme le souligne le conseiller de l'ambassade, Jacques Fouques-Duparc, cet accueil s'est fait en contradiction avec les engagements pris par la France, ce qui fragilise la position de la mission Vincent au moment de mener les négociations :

À la différence des asilés rangés sous les rubriques précédentes, les Espagnols, théoriquement en traitement et réfugiés en fait dans les hôpitaux de Madrid, [...] ne sont pas, au sens précis du terme, des asilés. Non seulement ils n'en ont pas la qualité reconnue, mais l'ambassade s'est même implicitement interdite de la revendiquer pour eux, quand, après accord du Département, elle a, au début de cette année, remis au ministère d'État des listes nominatives d'asilés, auxquelles elle a conféré un caractère limitatif et définitif, et dans lesquelles ils ne figurent pas. [...]

La fragilité particulière de cette situation me ferait hésiter à présenter à leur sujet la proposition transmise par M. Robert Vincent, quand bien même celle-ci ne me paraîtrait pas, dans sa rigueur, absolument imprésentable ${ }^{41}$.

C'est la précarité de la situation qui pousse les diplomates français à tirer avantage de la mission Chetwode pour éviter une négociation inconfortable. Lorsque la commission britannique obtient l'accord de principe de Barcelone et de Salamanque pour l'échange des " asilés » de toutes les ambassades et légations et sollicite les listes des personnes concernées, FouquesDuparc demande au consul de France à Madrid, Jacques Pigeonneau, un état nominatif des Espagnols hébergés dans les hôpitaux français. Il s'agit de mettre fin, une bonne fois pour toutes, à la question de l'asile diplomatique ${ }^{42}$. Le consul obtient ainsi du représentant de la commission, le colonel Cowen, que les Espagnols sous protection française soient inclus dans l'échange

39. Ibid., dép. n 570 de Fouques-Duparc au Quai d’Orsay, Barcelone, 4 décembre 1938

40. CADN/396PO/B/568, GC 4-C3, dép. nº 215 du Quai d’Orsay à Émile Lasmartres, Paris, 14 septembre 1938.

41. CADN/396PO/B/591, dép. nº 597 de Fouques-Duparc au Quai d'Orsay, Barcelone, 17 décembre 1938.

42. CADN, Consulat de France à Madrid, carton 87 (ci-après: CADN/397PO/1/87), tél. nº 60-61 de Fouques-Duparc à Jacques Pigeonneau, Barcelone, 30 octobre 1938. 
négocié ${ }^{43}$. Ce sont cependant la fin de la guerre civile et l'entrée des troupes franquistes à Madrid qui permirent aux Espagnols hébergés dans les hôpitaux français d'en sortir, avant que les négociations aient pu aboutir ${ }^{44}$.

D'autre part, les difficultés rencontrées par la mission Vincent poussent d'une certaine manière la diplomatie française à se désengager par rapport aux projets d'échanges. $\mathrm{Si}$, au moment de l'installation de la mission Chetwode, Labonne estime que " pour notre part, le déroulement de l'opération d'échange des asilés constitue un enseignement de nature à nous laisser perplexes ", et que les "chances de la mission d'échange elle-même sont incertaines", l'ambassade de France a tôt fait de reporter la charge de ces projets sur la mission britannique ${ }^{45}$. Ainsi, lorsque d'autres légations ou ambassades font appel aux bons offices de la France et aux contacts établis par la mission Vincent, les diplomates français préferent-ils les adresser à la mission Chetwode :

Je n'ai pas intentionnellement parlé dans cette lettre des asilés des autres ambassades et légations, qui se sont adressées à nos bons offices pour faciliter l'obtention d'un règlement. Je dois avouer, pour ma part, que je trouve notre tâche en la matière déjà assez lourde, et les résultats obtenus jusqu'ici trop incertains pour nous inciter à accepter délibérément de nouvelles missions, qui me paraissent du ressort de la commission Chetwode, plutôt que de cette ambassade ${ }^{46}$.

Enfin, parce qu'il ne s'agit pas de prétendre que le gouvernement français et son ambassade adoptent une simple posture opportuniste à l'égard de la mission Chetwode, il convient aussi de souligner que la France apporte ponctuellement son aide et son soutien à l'initiative britannique. C'est en tout cas l'engagement que prend Labonne auprès du ministre d'État Julio Álvarez del Vayo au moment de la visite du Field Marshall Chetwode à Barcelone ${ }^{47}$. Ainsi, lorsque l'ancien ministre basque Manuel de Irujo informe le conseiller de l'ambassade que le gouvernement républicain envisage, en ce qui concerne les exécutions, de mettre fin au sursis qu'il s'était engagé à respecter auprès de la commission britannique sous condition de réciprocité, le Quai d'Orsay charge son ambassadeur à Londres, Charles Corbin, d'en informer le Foreign Office et Chetwode ${ }^{48}$.

\section{CONCLUSION}

Les deux missions d'échange se retrouvent en concurrence sur le plan strictement humanitaire non par la volonté délibérée des gouvernements

43. CADN/397PO/1/87, tél. n 66-67 de Pigeonneau au Quai d'Orsay, Madrid, 22 février 1939

44. Ibid., tél. n 2 de Pigeonneau à Philippe Pétain, Madrid, 31 mars 1938.

45. CADN/378PO/C/235, 6-1-6, tél. n 654-655 de Labonne au Quai d’Orsay, Barcelone, 8 septembre 1938 .

46. CADN/396PO/B/591, dép. nº 597 de Fouques-Duparc au Quai d'Orsay, Barcelone, 17 décembre 1938.

47. CADN/378PO/C/235, 6-1-6, tél. nº 618 de Labonne au Quai d'Orsay, Barcelone, 22 août 1938

48. Ibid., tél. n² 2253 d'Émile Charvériat à Corbin, Paris, 21 novembre 1938. 
britannique et français de faire obstacle à l'une ou l'autre d'entre elles, mais du fait même de ce que l'on pourrait qualifier d'inflation humanitaire, qui a pour effet de compliquer encore davantage les négociations. Pour autant, les ambitions très différentes des deux missions leur permettent également de s'articuler de manière relativement complémentaire à l'occasion. C'est surtout vrai pour la mission Vincent dont les contours se limitent strictement à l'échange des "asilés » d'âge militaire inscrits sur les listes communiquées au gouvernement républicain. La mission Chetwode apparaît donc à celle-ci comme une opportunité pour pallier ses insuffisances.

C'est en revanche la visée politique dont est investie la mission Chetwode, les Britanniques en attendant sur le long terme la possibilité de développer une médiation politique en Espagne, qui est source de malentendu entre les deux pays, voire même de concurrence. En effet, les Britanniques fuient toute coordination internationale précisément pour préserver cette perspective et en récolter seuls les fruits, quand les Français - et tout particulièrement Labonne - cherchent à s'intégrer au processus. C'est que les deux pays ont quelque chose à gagner dans cette médiation politique en termes de prestige et de démonstration de puissance sur la scène internationale et devant l'opinion publique, mais aussi pour consolider leur position en Europe, vis-à-vis de l'Allemagne et de l'Italie notamment, et dans l'Espagne d'après-guerre. Or, l'Angleterre n'entend visiblement pas partager ces bénéfices, tandis que la France ne souhaite pas en être privée, bien qu'elle ne conçoive son intervention qu'en concertation avec l'Angleterre en raison de l'importance qu'elle accorde à l'alliance britannique.

D'une manière générale, c'est le phénomène d'inflation humanitaire en général qui peut être réinterprété à la lueur de la concurrence politique et internationale entre États. Finalement, dans le cas de la France et de la Grande-Bretagne, ces efforts humanitaires n'auraient-ils pas aussi comme objectif d'améliorer leur image auprès du camp nationaliste que, très tôt, elles considèrent comme le futur vainqueur de la guerre civile? $\mathrm{Ne}$ visent-ils pas à compenser le déficit de relations que les deux puissances entretiennent avec le général Franco ? La primauté des objectifs stratégiques britanniques sur les considérations humanitaires, qui, selon Peter Anderson, expliquerait le faible succès de la mission Chetwode, nous pousse à l'envisager ${ }^{49}$. On comprendrait dès lors plus aisément la multiplication des missions humanitaires, parfois concurrentes, ainsi que la très faible coordination internationale, chaque État étant porté à développer sa propre mission dans l'espoir d'en tirer les meilleurs fruits diplomatiques et politiques.

Nathan RousseLot Université de Nantes 
